\title{
Association between p16 Promoter Methylation and Thyroid Cancer Risk: A Meta-analysis
}

\author{
Wei Wu ${ }^{1}$, Sheng-Fu Yang ${ }^{2}$, Fei-Fei Liu ${ }^{1}$, Ji-Hong Zhang ${ }^{1 *}$
}

\begin{abstract}
Background: The aim of the meta-analysis was to derive a more precise assessment of the association between p16 promoter methylation and thyroid cancer risk. Materials and Methods: The PubMed, Web of Science databases and Chinese CNKI were searched for relevant articles. Ultimately, seventeen case-control studies were included with a total of 804 thyroid cancer cases and 487 controls analysis by $R$ Software ( $R$ version 3.1.2) including meta. Crude odds ratios with $95 \%$ confidence intervals were calculated using the random-effects model which were used to assess the strength of relationship between p16 methylation and lung carcinogenesis. Funnel plots were carried out to evaluate publication bias. Results: The meta-analysis results showed that the frequency of p16 promoter methylation in cancer tissue/blood was significantly higher than that normal tissue/ blood $(\mathrm{OR}=5.46,95 \% \mathrm{CI} 3.12-9.55, P<0.0001)$ by random effects model with small heterogeneity. Conclusions: Thus, p16 promoter methylation may be associated with thyroid cancer risk.
\end{abstract}

Keywords: Thyroid cancer - p16 promoter methylation - Meta-analysis

Asian Pac J Cancer Prev, 16 (16), 7111-7115

\section{Introduction}

Worldwide, thyroid cancer is among the less frequent malignancies. However, in the past decades the incidence has almost doubled-attributed for the most part to the increased diagnosis of papillary thyroid cancer (Wartofsky, 2010). While the reasons for the increase in thyroid cancer incidence require further elucidation, several lines of evidence indicate that in most circumstances the increase is attributable to optimal diagnostic scrutiny aided by technical advances (Sherman, 2003; Mazzaferri, 2012; Budak et al., 2013). Overall, the etiology of thyroid cancer is not fully understood. Currently, there has been a large progress in the molecular pathogenesis of thyroid cancer in recent years, which are well exemplified by the elucidation of several major signaling pathways and related molecular derangements of thyroid cancer (Yen et al., 2003; Goyal et al., 2013; Xing, 2013). Thyroid cancer has predominantly been considered a genetic disease, which is characterized by sequential accumulation of genetic alterations (Goyal et al., 2013; Xing, 2013). DNA methylation is a kind of genetic alterations regulation mechanism, which is known that DNA methylation is a major epigenetic modification in mammals, and changes in DNA methylation patterns play a key role in tumorigenesis in humans. In particular, promoter $\mathrm{CpG}$ island hypermethylation is closely related to inactivation and silencing, resulting in tumor suppressor loss of gene expression and X-chromosome inactivation, and affects the development of carcinogenesis (Baylin, 2005; Hesson et al., 2007). The p16 gene is known as a tumor suppressor gene which is may be involved in the development of thyroid cancer (Yang et al., 2012). It is localized on chromosome 9q21 and acts through the inhibition of CDK4/6 and thereby leaving the retinoblastoma $(\mathrm{RB})$ protein under-phosphorylated and consequently promoting cell cycle arrest (Ojima et al., 2006), but loss of p16 expression by its promoter methylation would lead to loss of control of the restriction point in the G1 phase of the cell cycle and favor cellular transformation (Esteller, 2008) and then lead to carcinogenesis. Over the past decade, there were many studies assessing the association between $\mathrm{p} 16$ promoter methylation and thyroid cancer risk, but the result is not clear. The aim of the meta-analysis was to derive a more precise assessment of the association between p16 promoter methylation and thyroid cancer risk.

\section{Materials and Methods}

\section{Literature search}

Relevant papers published before March 2015 was identified through a search of PubMed, Web of Science databases and Chinese CNKI using the following terms: ("thyroid neoplasm" or "thyroid cancer" or "thyroid

${ }^{1}$ Laboratory of Molecular Genetics of Aging \& Tumor, Faculty of Medicing, Kunming University of Science and Technology, ${ }^{2}$ Department of Pharmacy, The First People's Hospital of Yunnan Province, Kunming, Yunnan, China *For correspondence: zhjihong2000@126.com 
Wei Wu et al

carcinoma" ) and ("p16" or "cyclin-dependent kinase inhibitor 2" or "multiple tumor suppressor 1" or "MTS1" or "MTS1" or "CDKN2A" or "P16-INK4A" or "P16INK4A" or "TP16") and ("methylation" or "DNA methylation" or "hypermethylation"). The references of the eligible articles or reviews were also reviewed to check through manual searches to find other potentially studies.

\section{Inclusion and exclusion criteria}

The included articles meet the following criteria: (1) original study; (2) the diagnosis of thyroid cancer was based on histopathology; (3) the sample for the analysis was obtained from biopsy or surgical tumor tissue/ blood specimens; (4) studies were included if they had a case-control design and available frequency of the p16 promoter methylation; (5) when the same or overlapping data appeared in multiple publications, the most recent or largest population were used. To avoid duplication of data, we carefully checked the author names, research institutions and procedures for enrolling participants. Where several publications reported from the same population data, only the most rounded study with more information was included. Studies were excluded if they met the following criteria: (1) evaluating the associations of thyroid cancer with other gene; (2) case reports, letters, or reviews; (3) incomplete data or no usable data were reported; and (4) studies contained duplicate data.

\section{Data extraction}

Data was extracted independently from each study by two reviewers (W. Wu and Y.Z. Huang) using prespecified selection standards. Decisions were made and disagreements about study selection were resolved by consensus or by involving a third reviewer (S.F. Yang). The following information was extracted from the studies: the first author's last name, publication year, the country where patients received treatment, and numbers of cases and controls, the number of p16 promoter methylation individuals in each subgroups, etc.

\section{Statistical analysis}

The strength of the association between the p16 promoter methylation and thyroid cancer was measured by pooled odds ratio (OR) with its $95 \%$ confidence interval (CI).The significance of the pooled OR was determined by the $\mathrm{Z}$ test and $p<0.05$ was considered as statistically significant. Heterogeneity was tested using the $\mathrm{I}^{2}$ statistic with values over $50 \%$ and Chi-squared test with $\mathrm{P} \leq 0.1$ indicating strong heterogeneity between the studies (Higgins et al., 2003). Tau-squared ( $\tau 2$ ) was used to determine how much heterogeneity was explained by subgroup differences. The data were pooled using the DerSimonian and Laird random effects model $\left(\mathrm{I}^{2}>\right.$ $50 \%, \mathrm{P} \leq 0.1)$ or fixed effects model $\left(\mathrm{I}^{2}<50 \%\right)$ according to heterogeneity statistic $\mathrm{I}^{2}$ (DerSimonian and Laird,

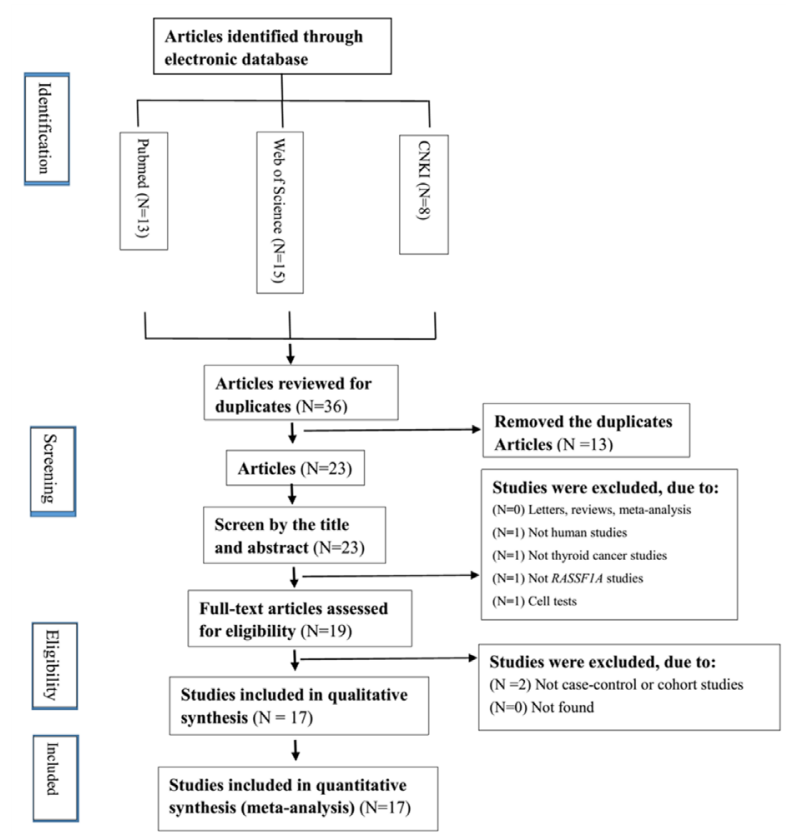

Figure 1. Flow Chart. The initial search strategy yielded 36 potential titles to be included in this review. Ultimately, 17 articles were selected

Table 1. Main Characteristics of the Studies Included in the Meta-analysis

\begin{tabular}{|c|c|c|c|c|c|c|c|c|c|c|c|}
\hline \multirow[t]{2}{*}{ Author } & \multirow[t]{2}{*}{ Year } & \multirow[t]{2}{*}{ Country } & \multirow[t]{2}{*}{ Method } & \multirow{2}{*}{ Sample } & \multirow{2}{*}{$\begin{array}{c}\text { Mean } \\
\text { age (year) }\end{array}$} & \multirow{2}{*}{$\begin{array}{l}\text { TNM. } \\
\text { Stage }\end{array}$} & \multirow[b]{2}{*}{ Male/Female } & \multicolumn{2}{|c|}{ Case } & \multicolumn{2}{|c|}{ Control } \\
\hline & & & & & & & & M & $\mathrm{T}$ & M & $\mathrm{T}$ \\
\hline Li et al. & 2013 & China & nMSP & Blood & 40 & I-IV & $15 / 35$ & 27 & 50 & 0 & 32 \\
\hline Brait et al. & 2012 & USA & QMSP & Tissue & 47 & I-IV & $10 / 33$ & 1 & 44 & 1 & 15 \\
\hline Dai et al. & 2012 & China & nMSP & Tissue & 40 & I-IV & $15 / 35$ & 27 & 50 & 5 & 32 \\
\hline Wang et al. & 2012 & China & MSP & Tissue & 46 & I-IV & $14 / 60$ & 20 & 74 & 0 & 21 \\
\hline Mohamamdiasl et al. & 2011 & Iran & COBRA & Tissue & 50 & NA & $7 / 18$ & 10 & 25 & 7 & 25 \\
\hline Dai et al. & 2010 & China & nMSP & Tissue & 40 & NA & $15 / 35$ & 27 & 50 & 5 & 32 \\
\hline Li et al. & 2008 & China & MSRE & Blood & NA & I-IV & NA & 9 & 60 & 0 & 30 \\
\hline Wiseman et al. & 2008 & Canada & IHC & Tissue & 46 & NA & $26 / 73$ & 55 & 98 & 5 & 100 \\
\hline Lam et al. & 2007 & Australia & MSP & Blood & 40 & I-IV & $9 / 35$ & 18 & 44 & 0 & 9 \\
\hline Schagdarsurengin et al. & 2006 & Germany & MSP & Tissue & 52.7 & I-IV & $15 / 23$ & 9 & 38 & 0 & 22 \\
\hline Nasr et al. & 2006 & USA & IHC & Tissue & NA & NA & NA & 20 & 51 & 5 & 57 \\
\hline Huang et al. & 2006 & China & MSRE & Tissue & 35.1 & NA & $13 / 47$ & 9 & 60 & 0 & 30 \\
\hline Hoque et al. & 2005 & USA & QMSP & Tissue & NA & NA & NA & 5 & 20 & 2 & 15 \\
\hline Peng et al. & 2006 & China & MSP & Tissue & 50 & I-IV & $9 / 16$ & 9 & 25 & 0 & 9 \\
\hline Boltze & 2003 & Germany & MSP & Tissue & 53.8 & I-IV & $24 / 35$ & 36 & 59 & 8 & 33 \\
\hline Schagdarsurengin et al. & 2002 & USA & MSP & Tissue & NA & I-IV & NA & 9 & 36 & 1 & 5 \\
\hline Elisei et al. & 1998 & USA & MSP & Tissue & NA & NA & NA & 7 & 20 & 6 & 20 \\
\hline
\end{tabular}

MSP, methylation specific polymerase chain reaction; QMSP, quantitative methylation specific polymerase chain reaction; nMSP,nested methylation specific PCR; COBRA,Combined bisulfite restriction analysis; MSRE, methylation sensitive restriction endonuclease; T, the total number of patients in each article; $\mathrm{M}$, the number of patients with methylation; NA, not available 

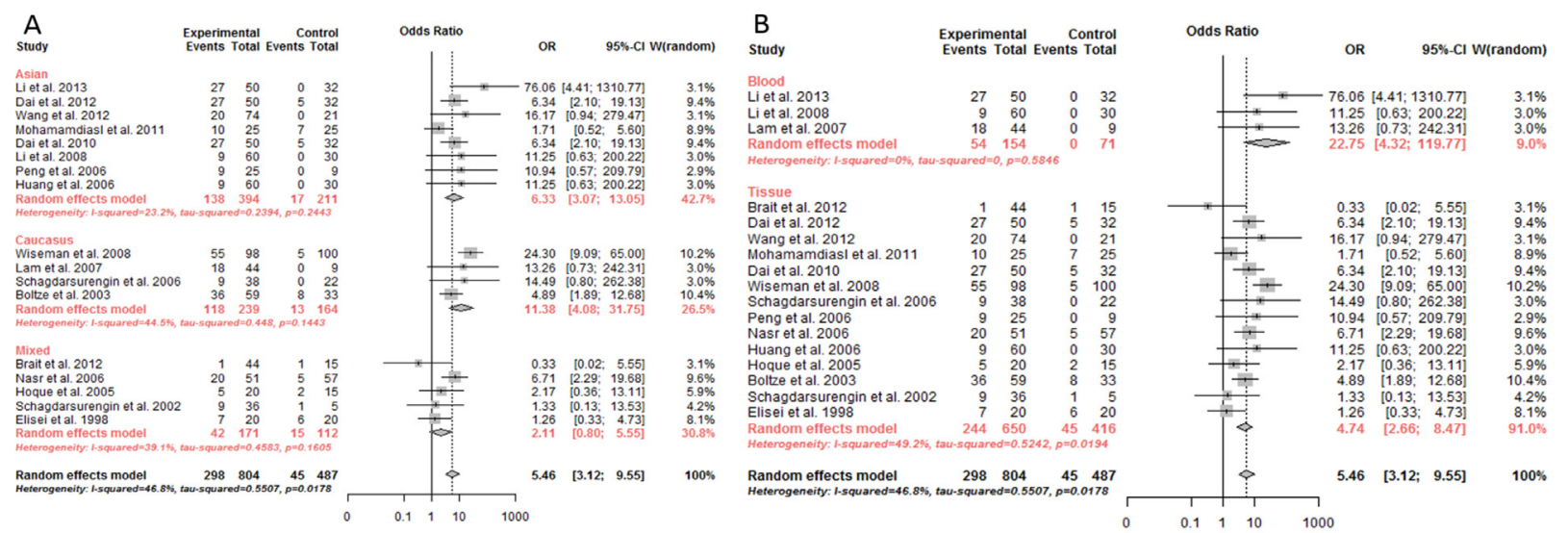

Figure 3. Subgroup Analysis by Ethnics and Sample for the Relationship between p16 Promoter Methylation and Thyroid Cancer Risk. A-B, The circles and horizontal lines correspond to the study specific odds ratio (OR) and $95 \%$ confidence interval (CI). The combined OR and $95 \% \mathrm{CI}$ are indicated by the diamonds

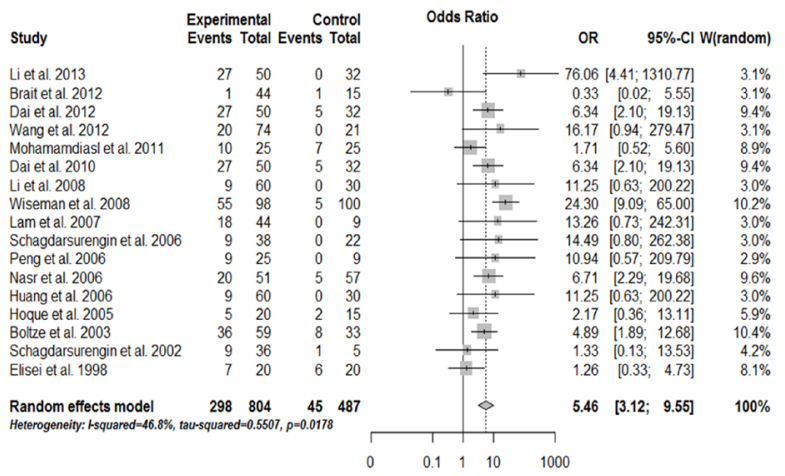

Figure 2. Meta-analysis for the Association between p16 Promoter Methylation and Thyroid Cancer Risk. The circles and horizontal lines correspond to the study specific odds ratio $(\mathrm{OR})$ and $95 \%$ confidence interval $(\mathrm{CI})$. The combined $\mathrm{OR}$ and $95 \% \mathrm{CI}$ are indicated by the diamonds

1986). With a lack of heterogeneity among included studies, the pooled odds ratio estimates were calculated using the fixed-effects model (Mantel and Haenszel, 1959). Otherwise, the random-effects model was used (DerSimonian and Laird, 1986). Sensitivity analyses were performed to assess the contributions of single studies to the final results. Begg's test were used to examine whether the results of a meta-analysis may have been affected by publication bias. Egger's test was implemented to testing for funnel plot asymmetry (M. Egger, 1997).All statistical analyses were performed using $\mathrm{R}$ Software $(\mathrm{R}$ version 3.1.2) including meta packages.

\section{Results}

Studies included in the meta-analysis according to the inclusion criteria, 17 case-control studies were included with a total of 804 thyroid cancer cases and 487 controls (Figure 1) (Elisei et al., 1998; Schagdarsurengin et al., 2002; Boltze et al., 2003; Hoque et al., 2005; Huang et al., 2006; Nasr et al., 2006; Peng et al., 2006; Schagdarsurengin et al., 2006; Lam et al., 2007; Li and He, 2008; Wiseman et al., 2008; Dai et al., 2010; Mohammadi-asl et al., 2011; Brait et al., 2012; Dai et al., 2012; Li et al., 2013; Wang et al., 2013). The characteristics of studies are listed in Table 1. The publication year of those eight studies ranged from 1998 to 2013, and the source of controls was mainly
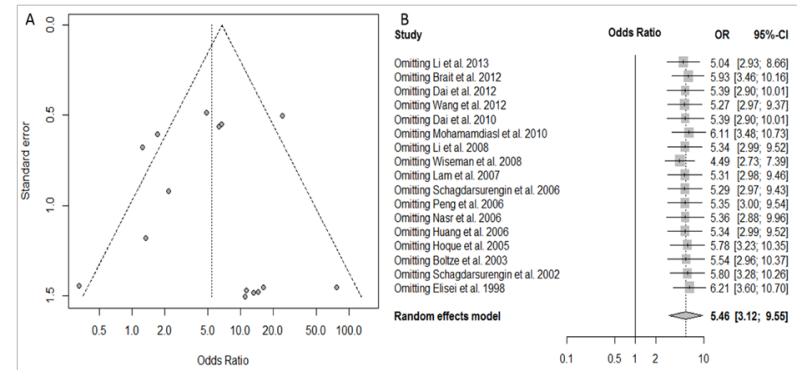

Figure 4. Funnel Plot for Publication Bias Test and Sensitivity Analysis on the Relationships between p16 Promoter Methylation and the Thyroid Cancer Risk. $\mathrm{A}$, The natural logarithm of odds ratio (OR) and its standard error were used in the funnel plot. The circles correspond to the $\log$ OR from individual trials, and the diagonal lines show the expected $95 \%$ confidence interval (CI) around the summary estimate. B, Sensitivity analysis of the summary odds ratio coefficients on the relationships between p16 promoter methylation and the thyroid cancer patients

based on healthy population or adjacent normal tissue. There were three studies from Caucasians, 5 studies from Mixed-race and 9 studies from Asians, 3 focus on blood sample and fourteen focus on tissue.

\section{Meta-analysis}

A summary of the meta-analysis of the association between 16 promoter methylation and thyroid cancer risk is provided in Figure 2. The meta-analysis results showed that the frequency of $\mathrm{p} 16$ promoter methylation in cancer tissue/blood was significantly higher than that normal tissue/blood $(\mathrm{OR}=5.46,95 \% \mathrm{CI}=[3.12 ; 9.55], \mathrm{z}=5.94$; $\left.\mathrm{P}<0.0001 ; \mathrm{I}^{2}=46.8 \% ; \tau 2=0.5507\right)$ by random effects model with small heterogeneity. Subgroup by ethnicity showed that there is a significant association between 16 promoter methylation and thyroid cancer risk in Caucasians, Asians and Mixed-race (Figure 3A). For subgroup by different sample, our meta-analysis result indicate that a significant result in blood sample and tissue sample when compare to the normal (Figure 3B).

\section{Publication bias and Sensitive Analysis}

Funnel plot was used to assess the publication bias of included studies in the meta-analysis. The shape 
of the funnel plots did not reveal any evidence of obvious asymmetry under all studies (Figures 4A). Sensitive analyses were conducted to determine whether modification of the inclusive criteria of the meta-analysis affected the final results. When we analysis the studies and found no difference between the 17 studies, indicating that our results were statistically robust (Figure 4B).

\section{Discussion}

P16 is regarded as a cell-cycle regulator which induces G1-phase arrest through inhibiting cyclin D dependent protein kinase 4 (CDK4) and 6 (CDK6), and considered as a key role for the growth, invasion, and metastasis of human cancers (Rayess et al., 2012). It has been demonstrated that P16 inactivation would damage the regulatory mechanism, and would thereby give rise to the promotion of carcinogenesis through multiple downstream transcriptional pathways. Many previous studies suggested that p16 promoter methylation was closely related to a great proportion of tumors including thyroid cancer (Schagdarsurengin et al., 2002; Boltze et al., 2003; Schagdarsurengin et al., 2006). Considering that the $\mathrm{CpG}$ island high methylation in the promoter region of tumor-suppressor genes is suspected of participating in the pathogenesis and progression of thyroid cancer, hence, the frequently methylated of p16 may use as a biomarker offering a new approach to ensure the early diagnosis of thyroid cancer.

In order to evaluate the exact role of p16 promoter methylation in lung carcinogenesis risk, we conducted a meta-analysis of 17 clinical studies with a total of 804 thyroid cancer cases and 487 controls. Our meta-analysis results revealed that the frequencies of p16 promoter methylation in the thyroid cancer patients were higher than those of normal $(\mathrm{OR}=5.46,95 \% \mathrm{CI} 3.12-9.55$, $\mathrm{P}<0.0001)$ by random effects model, suggesting that promoter methylation of p16 gene may play a key role in the thyroid carcinogenesis risk. Next, we stratified the association between 16 promoter methylation and thyroid cancer risk by ethnics and testing materials of thyroid cancer. Subgroup analysis by ethnics showed similar OR values in mixed-race, Asians and Caucasians, indicating a parallel effect of p16 promoter methylation on thyroid cancer risk among different ethnics. Moreover, we found that the ORs were also significant in subgroups with different testing materials of thyroid cancer. The sensitive analysis confirmed that there was no change in the odds ratios and the $95 \%$ confidence interval when altering the included studies; the publication bias supported the robust conclusion. Taken together, these results indicated a credible association of p 16 promoter methylation with thyroid cancer risk.

There are several limitations needed to be addressed in the meta-analysis. Firstly, in the subgroup analysis of Caucasians and blood, there were only three studies from Caucasians and two studies from blood, and the total sample size was still small. Therefore, more studies with a large number of participants are needed to further assess the association in Caucasians and blood, or in other race and sample. Secondly, our meta-analysis was based on unadjusted estimates because not all published studies provided adjusted estimates. Finally, although all thyroid cancer cases and controls of those studies were well defined with similar inclusion criteria, there may be potential factors that were not taken into account that may have some influence on the findings from the meta-analysis. Therefore, a meta-analysis including more high-quality designed epidemiology studies and a stratified analysis targeting different status are necessary in the future in this field.

In summary, this meta-analysis indicates that p 16 promoter methylation may be associated with thyroid cancer risk. Besides, more high-quality designed epidemiology studies with large numbers of participants are needed to further assess the association above.

\section{Acknowledgements}

This work was financially supported by the National Science Foundation of China (No.81260501, U1202221, U1132604).

\section{References}

Baylin SB (2005). DNA methylation and gene silencing in cancer. Nat Clin Pract Oncol, 2, -11.

Boltze C, Zack S, Quednow C, et al (2003). Hypermethylation of the CDKN2/p16INK4A promotor in thyroid carcinogenesis. Pathol Res Pract, 199, 399-404.

Brait M, Loyo M, Rosenbaum E, et al (2012). Correlation between BRAF mutation and promoter methylation of TIMP3, RARbeta2 and RASSF1A in thyroid cancer. Epigenetics, 7, 710-9.

Budak A, Gulhan I, Aldemir OS, et al (2013). Lack of influence of pregnancy on the prognosis of survivors of thyroid cancer. Asian Pac J Cancer Prev, 14, 6941-3.

Dai YL, Cai DH, Chen H, et al (2012). [The relevance between the promoter hypermethylation of tshr and p16 gene and clinicopathological parameters in human papillary thyroid carcinoma]. J Capital Medical University, 33, 361-5

Dai YL, Zhang F, Ye J, et al (2010). [P16 promoter hypermethylation in human papillary thyroid carcinoma]. Guangdong Medical Journal, 31, 1804-7.

DerSimonian R, Laird N (1986). Meta-analysis in clinical trials. Control Clin Trials, 7, 177-88.

Elisei R, Shiohara M, Koeffler HP, et al (1998). Genetic and epigenetic alterations of the cyclin-dependent kinase inhibitors p15INK4b and p16INK4a in human thyroid carcinoma cell lines and primary thyroid carcinomas. Cancer, 83, 2185-93.

Esteller M (2008). Epigenetics in cancer. $N$ Engl J Med, 358, 1148-59.

Goyal N, Setabutr D, Abdulghani J, et al (2013). Molecular and genetic markers of follicular-cell thyroid cancer: etiology and diagnostic and therapeutic opportunities. Adv Exp Med Biol, 779, 309-26.

Hesson LB, Cooper WN, Latif F (2007). The role of RASSF1A methylation in cancer. Dis Markers, 23, 73-87.

Higgins JP, Thompson SG, Deeks JJ, et al (2003). Measuring inconsistency in meta-analyses. BMJ, 327, 557-60.

Hoque MO, Rosenbaum E, Westra WH, et al (2005). Quantitative assessment of promoter methylation profiles in thyroid neoplasms. J Clin Endocrinol Metab, 90, 4011-8.

Huang P, Li DX, Zhang Y (2006). [Methylation of p16 gene and expression of $\mathrm{p} 16$ protein in human thyroid neoplasms]. $J$ 
Practical Oncology, 21, 49-52

Lam AK, Lo CY, Leung P, et al (2007). Clinicopathological roles of alterations of tumor suppressor gene p16 in papillary thyroid carcinoma. Ann Surg Oncol, 14, 1772-9.

Li JJ, He Y (2008). [Expression and Alterations of p16 Gene in Human Primary Thyroid Neoplasm]. Progress in Modern Biomedicine, 8, 1253-5.

Li XF, Jin YL, He ZL, et al (2013). [Detecting the abnormal methylation of plasma 16 promoter in patients with thyroid carcinoma by nested-methylation-specific polymerase chain reaction]. Chinese Journal of Prevention and Control of Chronic Diseases, 21, 34-6.

M. Egger GDS, M. Schneider, and C. Minder (1997). Bias in meta-analysis detected by a simple, graphical test. BMJ Open.

Mantel N, Haenszel W (1959). Statistical aspects of the analysis of data from retrospective studies of disease. J Natl Cancer Inst, 22, 719-48.

Mazzaferri EL (2012). Managing thyroid microcarcinomas. Yonsei Med J, 53, 1-14.

Mohammadi-asl J, Larijani B, Khorgami Z, et al (2011). Qualitative and quantitative promoter hypermethylation patterns of the P16, TSHR, RASSF1A and RARbeta2 genes in papillary thyroid carcinoma. Med Oncol, 28, 1123-8.

Nasr MR, Mukhopadhyay S, Zhang S, et al (2006). Immunohistochemical markers in diagnosis of papillary thyroid carcinoma: Utility of HBME1 combined with CK19 immunostaining. Mod Pathol, 19, 1631-7.

Ojima H, Saito K, Yamauchi H, et al (2006). P16 protein abnormality in Epstein-Barr virus-associated gastric carcinomas. Anticancer Res, 26, 933-7.

Peng ZL, Cao RX, Wen GB, et al (2006). [The methylation of p16 gene in papillary thyroid carcinoma]. J Modern oncology, 14, 1501-3

Rayess H, Wang MB, Srivatsan ES (2012). Cellular senescence and tumor suppressor gene p16.Int J Cancer, 130, 1715-25.

Schagdarsurengin U, Gimm O, Dralle H, et al (2006). CpG island methylation of tumor-related promoters occurs preferentially in undifferentiated carcinoma. Thyroid, 16, 633-42.

Schagdarsurengin U, Gimm O, Hoang-Vu C, et al (2002). Frequent epigenetic silencing of the $\mathrm{CpG}$ island promoter of RASSF1A in thyroid carcinoma. Cancer Res, 62, 3698-701.

Sherman SI (2003). Thyroid carcinoma. Lancet, 361, 501-11.

Wang P, Pei R, Lu Z, et al (2013). Methylation of p16 CpG islands correlated with metastasis and aggressiveness in papillary thyroid carcinoma. J Chin Med Assoc, 76, 135-9.

Wartofsky L (2010). Increasing world incidence of thyroid cancer: increased detection or higher radiation exposure? Hormones (Athens), 9, 103-8.

Wiseman SM, Melck A, Masoudi H, et al (2008). Molecular phenotyping of thyroid tumors identifies a marker panel for differentiated thyroid cancer diagnosis. Ann Surg Oncol, 15, 2811-26.

Xing M (2013). Molecular pathogenesis and mechanisms of thyroid cancer. Nat Rev Cancer, 13, 184-99.

Yang D, Zhang H, Hu X, et al (2012). Abnormality of pl6/ p38MAPK/p53/Wipl pathway in papillary thyroid cancer. Gland Surg, 1, 33-8.

Yen TW, Shapiro SE, Gagel RF, et al (2003). Medullary thyroid carcinoma: results of a standardized surgical approach in a contemporary series of 80 consecutive patients. Surgery, 134, 890-9. 